\title{
Evaluating Amazon's Mechanical Turk for psychological research on the symbolic control of attention
}

\author{
Joseph R. Pauszek ${ }^{1}$ - Pedro Sztybel $^{1}$ • Bradley S. Gibson ${ }^{1,2}$
}

Published online: 26 January 2017

(C) Psychonomic Society, Inc. 2017

\begin{abstract}
The use of online crowdsourcing services like Amazon's Mechanical Turk (AMT) as a method of collecting behavioral data online has become increasingly popular in recent years. A growing body of contemporary research has empirically validated the use of AMT as a tool in psychological research by replicating a wide range of well-established effects that have been previously reported in controlled laboratory studies. However, the potential for AMT to be used to conduct spatial cuing experiments has yet to be investigated in depth. Spatial cuing tasks are typically very basic in terms of their stimulus complexity and experimental testing procedures, thus making them ideal for remote testing online that requires minimal task instruction. Studies employing the spatial cuing paradigm are typically aimed at unveiling novel facets of the symbolic control of attention, which occurs whenever observers orient their attention through space in accordance with the meaning of a spatial cue. Ultimately, the present study empirically validated the use of AMT to study the symbolic control of attention by successfully replicating four hallmark effects reported throughout the visual attention literature: the leftright advantage, cue type effect, cued axis effect, and cued endpoint effect. Various recommendations for future endeavors using AMT as a means of remotely collecting behavioral data online are also provided. In sum, the present study provides a crucial
\end{abstract}

Electronic supplementary material The online version of this article (doi:10.3758/s13428-016-0847-5) contains supplementary material, which is available to authorized users.

Bradley S. Gibson

bgibson@nd.edu

University of Notre Dame, Notre Dame, IN, USA

2 Department of Psychology, University of Notre Dame, 118 Haggar Hall, Notre Dame, IN 46556, USA first step toward establishing a novel tool for conducting psychological research that can be used to expedite not only our own scientific contributions, but also those of our colleagues.

Keywords Amazon · Mechanical Turk · Crowdsourcing • Visual attention $\cdot$ Spatial cuing

The life of a researcher is often a roller coaster of emotions. On the one hand, conducting research that unveils novel insights into the human mind and body can be extremely fulfilling in terms of one's own intellectual and professional development. However, on the other hand, it is easy to be discouraged by the practical limitations associated with collecting behavioral data. Typically, researchers pour an immeasurable amount of time and effort into developing and creating experiments that are both internally valid and externally significant. Then, the next few weeks or months are spent collecting enough data to detect the anticipated effects, with little or no theoretical progress made during that time. As Crump, McDonnell, and Gureckis (2013) so aptly described, "progress depends on the ebb and flow of the semester." This issue is even more salient to those researchers who collect data at smaller universities with restricted participant pools, and to those researchers who desire to extend the implications of their findings beyond the conventional undergraduate, who is Western, educated, industrialized, rich, and democratic (i.e., WEIRD; see Henrich, Heine, \& Norenzayan, 2010).

At least three broad issues are currently associated with the way in which psychological research is normally conducted. The first issue is the necessary reliance on undergraduate college students as research participants, even though "college students are hardly a random sample of all humanity" (Myers, 1983). The second issue is the plodding nature of data collection. A hypothesis is only as strong as the data that support it, 
and the contribution of any given researcher to a field of study often mirrors the rate at which the researcher can collect enough data to support his or her claims. As a result, researchers who collect data at large universities with an abundance of potential participants have an unspoken advantage over others. The third issue is with regard to obtaining pilot data from small-scale preliminary testing conducted in an effort to evaluate the practicality, time, cost, adverse events, and effect size associated with the intended experiment or study. In some cases, obtaining pilot data can be quite tedious, effortful, and time-consuming, even when only a small pool of pilot participants are required.

Fortunately, the growing popularity of online crowdsourcing services has offered a feasible solution to all three of the abovementioned issues associated with psychological research. In recent years, the most popular online crowdsourcing service used by psychologists for collecting behavioral data has been Amazon's Mechanical Turk (AMT; www.mturk.com). Although it is primarily used to collect short reviews of a variety of goods and services, AMT also allows researchers to conduct behavioral experiments over the Internet with participants drawn from a large and diverse pool that more accurately reflects the diversity of all humanity. Online crowdsourcing services like AMT also allow researchers to effectively "buy time" and exchange monetary compensation for rapid subject participation over the Internet, thereby substantially reducing the duration of stagnant periods otherwise devoted to data collection in the laboratory. As we will discuss in more detail in a later section, online data collection methods certainly have their shortcomings. For instance, they open the possibility of participant deception, and the testing environment cannot be controlled, or even known, when data are collected remotely. Nonetheless, AMT offers researchers "a new source of inexpensive, yet high-quality data" (Buhrmester, Kwang, \& Gosling, 2011) that has the potential to completely revolutionize the nature of data collection and psychological research as a whole. Therefore, it is worthwhile for researchers to more closely investigate the viability of online crowdsourcing services like AMT for conducting research within their respective fields of study. In so doing, they can provide empirical support for an innovative method of data collection that can expedite not only their own scientific contributions, but also those of their colleagues.

\section{The basics of AMT}

A number of recent publications have provided in-depth overviews regarding how AMT can be properly used to conduct behavioral experiments online (see, e.g., Bates \& Lanza, 2013; Buhrmester, Kwang, \& Gosling, 2011; Crump, McDonnell, \& Gureckis, 2013; Enochson \& Culbertson,
2015; Gibson, Piantadosi, \& Fedorenko, 2011; Mason \& Suri, 2012; Paolacci, Chandler, \& Ipeirotis, 2010; Woods, Velasco, Levitan, Wan, \& Spence, 2015). Thus, for the sake of brevity, the discussion of the basics of AMT provided in this section will be fairly succinct. Broadly, AMT allows requesters (i.e., researchers) to post human intelligence tasks (HITs) that are completed by workers (i.e., participants) in exchange for monetary compensation. The workforce provided by AMT is quite large and diverse. For instance, as of 2014, AMT was reported to house over 500,000 active workers, drawn from over 190 countries, who complete thousands of HITs on a daily basis (Paolacci \& Chandler, 2014). If they so desire, requesters can select any number of prespecified worker qualifications in an effort to restrict the pool of potential workers to a unique subsect of AMT's workforce. The worker qualifications that are appropriate for any given study obviously vary from experiment to experiment. Accordingly, requesters are also permitted to create and select their own custom worker qualifications as they see fit.

Although the majority of HITs on AMT require workers to provide brief reviews of websites, images, or writing samples, a growing number of full-length cognitive experiments have also been posted in recent years. The AMT website offers a variety of different project design templates to requesters, allowing them to create basic surveys or write requests for reviews through an intuitive "drag-and-drop" interface. If necessary, requesters can also adjust these versatile templates by modifying the HTML computer code of the HIT webpage. Alternatively, if the particular software needed to run an experiment cannot be directly incorporated into the HIT itself (e.g., because most websites do not permit links to selfextracting experimental software applications), requesters can use the HIT webpage to redirect workers to an alternative file-hosting service like Dropbox (www.dropbox.com). The requester can then provide workers with an experimental link on the file-hosting service that workers can temporarily download onto their computers. This latter method was used in the present study, and an overview of this process is provided in the Supplementary Materials for those interested.

\section{Empirical validation through replication}

A growing number of recent studies have empirically validated the use of AMT as a viable data collection method by replicating a wide range of well-established effects in acceptability and similarity judgment tasks (Gibson, Piantadosi, \& Fedorenko, 2011; Schnoebelen \& Kuperman, 2010; Sprouse, 2011), sentence completion tasks (Fine, Jaeger, Farmer, \& Qian, 2013; Schnoebelen \& Kuperman, 2010), artificiallanguage learning tasks (Becker, Nevins, \& Levine, 2012), psycholinguistic tasks (Enochson \& Culbertson, 2015), and basic visual attention and involuntary attention control tasks 
(Crump, McDonnell, \& Gureckis, 2013). However, relatively less is known about the replicability of certain hallmark effects found in studies of the symbolic control of attention. The symbolic control of attention is said to occur whenever an observer covertly orients attention in accordance with the meaning of a spatial cue. The spatial cues most frequently used to study the symbolic control of attention in the laboratory are spatial words (e.g., "Above," "Below," "Left," and "Right") and arrows (e.g., $\uparrow, \downarrow, \leftarrow$, and $\rightarrow$ ), which likely reflects the ubiquity of these stimuli in everyday life (see Gibson \& Sztybel, 2014, for a review). As a result, the present study focused on replicating a variety of effects associated with the processing of spatial word cues and arrow cues in particular, in addition to an underlying spatial bias that is thought to induce a measurement bias in a commonly used experimental paradigm. Brief overviews of each of the hallmark effects of interest in the present study will be provided in subsequent sections.

Prior to using AMT to uncover new facets of the symbolic control of attention, it was first necessary to establish the replicability of these abovementioned effects so that they could be accounted for in all statistical analyses. This was necessary because contemporary research has reported that, when they are left uncontrolled, these effects have the capacity to bias commonly used measures of cue comprehension that rely on comparisons between the mean correct response times (RTs) on cued and uncued trials (i.e., costs-plus-benefits; see Pauszek \& Gibson, 2016; Posner, 1980; Posner, Snyder, \& Davidson, 1980). If unaccounted for, this measurement bias can lead to potentially false inferences about the nature of attention control, which will clearly be an important issue to consider in virtually all future spatial cuing studies (see Pauszek \& Gibson, 2016, for a broader discussion of this issue). Therefore, in the present study we aimed to provide a crucial first step toward opening new avenues for efficiently and reliably investigating the symbolic control of attention and, more generally, visual attention as a whole.

\section{Important effects in the spatial cuing literature}

Over the past 30 years, the most common experimental paradigm used to study the symbolic control of attention has been the spatial cuing paradigm (Posner, 1980; Posner et al., 1980; see also Gibson \& Sztybel, 2014, for a review). In this paradigm, observers are required to comprehend and use a spatial cue to covertly orient their attention toward some to-bedetected or to-be-discriminated target stimulus in the visual periphery. Spatial cuing tasks are typically very basic in terms of their stimulus complexity and experimental testing procedures, thus making them ideal for remote testing online that requires minimal task instruction.
The simplicity of the spatial cuing paradigm is certainly advantageous in terms of controlling for confounds and isolating the effects of experimental manipulations. However, as mentioned, a growing body of evidence suggests that measurement of observers' performance in the spatial cuing paradigm is sensitive to a variety of factors that are orthogonal to the task itself (see, e.g., Nomura, Katahata, \& Hashiya, 2005; Pauszek \& Gibson, 2016). As a result, is it necessary to consider the possibility that the effects of interest in the present study may not replicate due to some unforeseen confound associated with collecting data remotely over the Internet. Examples of such potential confounds include, but are not limited to, a lack of control over the fine calibrations (e.g., resolutions and refresh rates) of workers' personal computers used for testing and an increased likelihood of distraction surrounding the worker during testing. Another prominent concern when collecting data remotely over the Internet is the potential for scammers to deceive researchers by utilizing non-human, pseudo-worker computer programs (i.e., bots) as a means of receiving compensation while subverting authentic participation. Thus, if the effects of interest in the present study are not found to replicate when the data are collected online, they will need to be more closely scrutinized in an effort to determine whether remote testing is simply an unstable data collection method or, even more troubling, whether the effects are merely artifacts of some presently unknown extraneous factors associated with collecting data in a controlled laboratory setting.

The primary aim of the present study was therefore to validate AMT as a tool for cognitive behavioral research, with a focus on the spatial cuing paradigm and four hallmark effects reported in previous studies of the symbolic control of attention. If the behavioral data obtained online were found to replicate these effects with reasonable fidelity, it would empirically validate the use of AMT as an innovative new data collection method that can be adopted with confidence in future studies of attention control. In particular, through the present study we attempted to extend the ongoing record of successful replications to four effects reported throughout the attention literature: (1) the left/right advantage, (2) the cue type effect, (3) the cued axis effect, and (4) the cued endpoint effect. Brief overviews of each of these effects are provided, in turn, in the following sections.

\section{The left/right advantage}

The left/right advantage is thought to reflect the asymmetrical organization of the human visual system (Abrams, Nizam, \& Carrasco, 2012; Carrasco, 2014; Kobayashi \& Kohshima, 1997, 2001a, b; Nomura, Katahata, \& Hashiya, 2005; Ossandón, Onat, \& König, 2014; Pauszek \& Gibson, 2016; see also Robertson, 2004, for a review). In the context of the 
spatial cuing paradigm, the left/right advantage manifests as faster RTs due to more efficient target processing when the target appears at the left or right location, relative to when the target appears at the above or below location. This effect is thought to arise from the abundance of cones and ganglion cells (Curcio \& Allen, 1990; Curcio, Sloan, Kalina, \& Hendrickson, 1990; Curcio, Sloan, Packer, Hendrickson, \& Kalina, 1987), parvocellular layers of the lateral geniculate nuclei in the thalamus (Connolly \& Van Essen, 1984), and cortical tissue in primary visual cortex (i.e., Area V1; Tootell, Switkes, Silverman, \& Hamilton, 1988; Van Essen, Newsome, \& Maunsell, 1984) and in the middle temporal visual area (i.e., Area MT; Maunsell \& Van Essen, 1987) that are more densely organized along the horizontal axis, relative to the vertical axis, of observers' visual field. Using the spatial cuing task depicted in Fig. 1a, Pauszek and Gibson

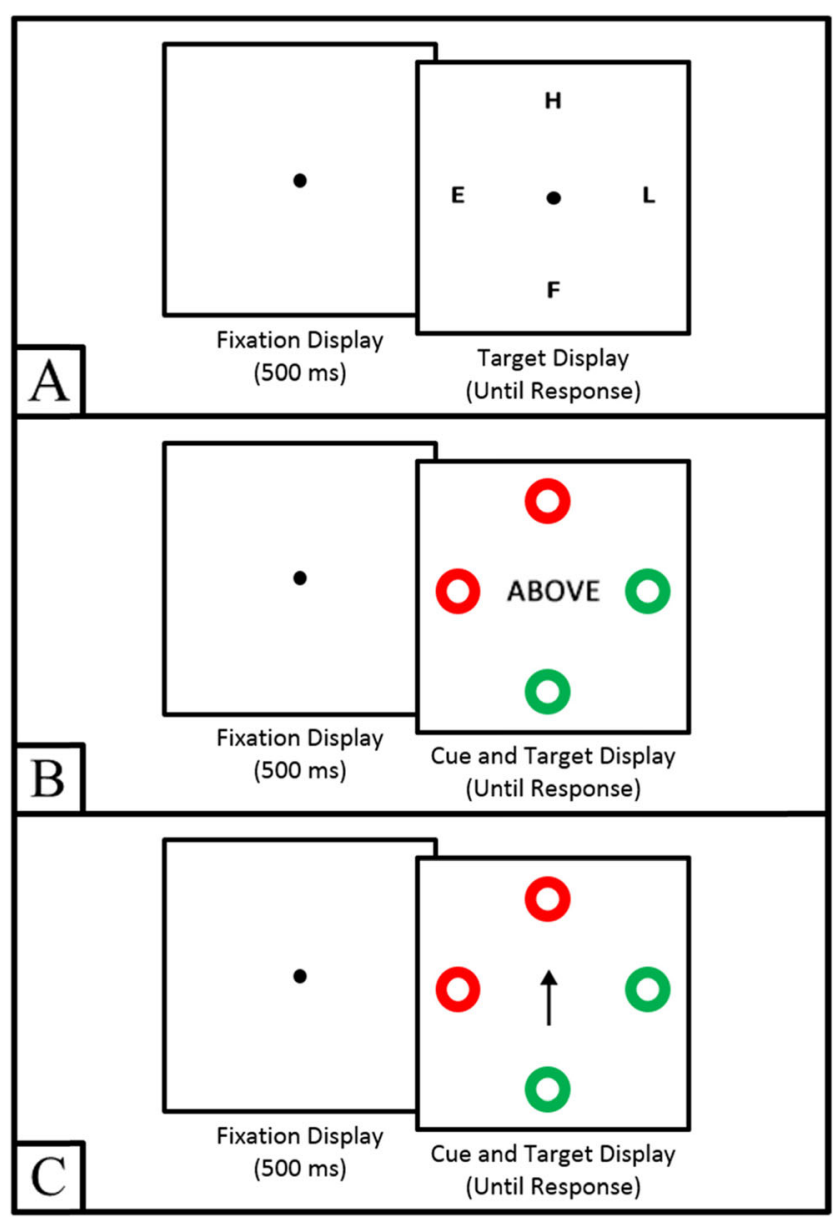

Fig. 1 Typical display sequences used in the present study. Panel a depicts a typical display sequence in $\mathrm{Block}_{\mathrm{nc}}$, during which workers were required to locate and discriminate the identity $(H$ or $U$ ) of the target letter present in each display. Panels $b$ and $c$ depict typical display sequences in $\mathrm{Block}_{\mathrm{w}}$ and Block $\mathrm{a}$, respectively, during which workers were required to discriminate the color (red or green) of the target circle indicated by the spatial cue. Note that the orders of Block and Block $\mathrm{a}_{\mathrm{a}}$ were counterbalanced across workers; however, all workers completed Block $_{\mathrm{nc}}$ first. The figure is not drawn to scale
(2016) provided evidence that an underlying left/right advantage operates in the spatial cuing paradigm, even when observers are not provided with a spatial cue. Moreover, the authors reported that the effect operates in an equipotent fashion along the horizontal axis (i.e., no differences were found between RTs or percent error rates when the target appeared at the left or right locations) and that it has the capacity to bias commonly used measures of cue comprehension if left uncontrolled (see Pauszek \& Gibson, 2016, for a broader discussion of this issue). Thus, establishing the replicability of the left/right advantage when remotely collecting data online was a necessary first step so that it can be controlled for in all future endeavors.

\section{The cue type effect}

The cue type effect is thought to reflect differences in the cognitive processing required to comprehend various types of spatial cues. For instance, the comprehension of spatial word cues is thought to require the computation of spatial reference frame parameters, thus making them relatively more effortful and time-consuming to process and use; arrow cues, on the other hand, are not thought to require these computations, thus making them relatively less effortful and timeconsuming to process and use (Gibson \& Kingstone, 2006; Gibson, Scheutz, \& Davis, 2009; Logan, 1995). Using the spatial cuing tasks depicted in Fig. 1b and c, Gibson and Kingstone showed that this effect manifests as faster overall RTs when the relatively easy-to-comprehend arrow cues are used than when the relatively difficult-to-comprehend spatial word cues are used (see Logan, 1995, for a broader discussion of the computation of spatial reference frame parameters; see also Kemmerer, 2006).

\section{The cued axis effect}

The cued axis effect is thought to reflect the support, or lack thereof, that the computation of spatial reference frame parameters receive from environmental regularities like gravity (Gibson \& Kingstone, 2006; Gibson et al., 2009; Kemmerer, 2006; Logan, 1995). Using the spatial cuing tasks depicted in Fig. $1 \mathrm{~b}$ and c, Gibson and Kingstone showed that this effect manifests as faster RTs when the spatial word cues "Above" or "Below" are used, relative to when the spatial word cues "Left" or "Right" are used (see also Gibson et al., 2009; Kemmerer, 2006; Logan, 1995). As we previously alluded to, the comprehension of relatively easy-to-comprehend arrow cues do not require the computation of spatial reference frame parameters. As a result, the cued axis effect (i.e., faster RTs when the cue refers to a target location along the vertical axis, as opposed to when the cue refers to a target location along the 
horizontal axis) is expected to be observed when spatial word cues, but not when arrow cues, are processed and used to orient attention toward the target.

\section{The cued endpoint effect}

The cued endpoint effect is thought to reflect the narrower focus of attention elicited when the spatial word cues "Above" or "Below" are used, relative to when the spatial word cues "Left" or "Right" are used (Gibson et al. 2009). In their series of experiments using the spatial cuing tasks depicted in Fig. 1b and c, Gibson et al. (2009) observed that, on any given trial, the color of the cued target circle and the color of the circle at the opposite endpoint of the cued target axis could be either compatible (i.e., the same color) or incompatible (i.e., the opposite color). When the spatial word cues "Left" or "Right" were used, those researchers observed that RTs were significantly slower when the target and the opposite distractor were incompatible than when they were compatible. However, this difference was not found when the spatial word cues "Above" or "Below" were used, nor when arrow cues were used (Gibson et al., 2009). Ultimately, this pattern of results was attributed to previous experience with inconsistent mappings between certain spatial symbols and absolute directions. For instance, words like "Left" and "Right" can refer to the opposite direction when encountered from an opposing perspective, whereas words like "Above" and "Below" or arrows tend to refer to the same absolute direction, regardless of the observers' perspective. Therefore, arrow cues and the spatial word cues "Above" and "Below" are thought to elicit a narrower focus of attention than the spatial word cues "Left" and "Right." Accordingly, in the present study, the compatibility or incompatibility of the target circle and the opposite distractor was expected to have a significant effect on RTs when spatial word cues referring to the left or right target locations were used, but not when spatial word cues referring to the above or below target locations were used, nor when arrow cues were used (Gibson et al., 2009).

\section{The present study}

The purpose of the present study was to validate AMT as a viable option for remotely conducting visual attention experiments using the spatial cuing paradigm, which requires fine temporal control of stimulus presentation and response recordings. In particular, with the present study we aimed to replicate four important effects that have been reported in recent investigations of spatial cuing and the semantics of space: the left/right advantage, the cue type effect, the cued axis effect, and the cued endpoint effect. If these effects were found to successfully replicate using a remote data collection method with minimal experimental control, it would support the notion that the effects indeed arise from their supposed origins and that AMT is a viable option to be used in future endeavors. However, if any or all of these effects were not found to replicate, it would be possible that they were not detectable through AMT due to the myriad of unpredictable factors associated with remotely collecting data online. An alternative, and even more troubling, conclusion arising from a failure to replicate would be that the effects were merely artifacts of some currently unknown extraneous factors associated with data collection in a controlled laboratory setting. Obviously, the latter conclusion would raise a number of concerns regarding the conclusions derived from the observation of these effects in the laboratory. Thus, the present study was expected to make a substantial contribution to the literature by either empirically validating a novel method of data collection, or by suggesting that the methodology of such experiments ought to be more closely scrutinized for potentially eliciting superfluous effects.

\section{Overview of the experimental blocks}

In an effort to investigate the aforementioned effects of interest in the present study, three experimental blocks were created; each was a different variation of the spatial cuing paradigm (Posner, 1980; Posner, Snyder, \& Davidson, 1980). In particular, workers were required to complete three variants of a spatial cuing task using no cue, a spatial word cue, and an arrow cue. For the sake of brevity, these experimental blocks will hereafter be referred to, respectively, as Block $\mathrm{nc}_{\mathrm{nc}}$, Block $\mathrm{w}_{\mathrm{w}}$, and Block $\mathrm{a}_{\mathrm{a}}$ In Block $\mathrm{nc}$, workers were required to locate and discriminate the identity ( $H$ or $U$ ) of a target letter presented alongside three visually similar, nontarget distractor letters in each target display (see Fig. 1a). This task was intended to replicate Experiment 1 of Pauszek and Gibson's (2016) study, which reported a left/right advantage. In Block $\mathrm{w}_{\mathrm{w}}$ and Block $\mathrm{a}_{\mathrm{a}}$, workers were required to discriminate the color (red or green) of a target circle specified by either a spatial word cue (see Fig. 1b) or an arrow cue (see Fig. 1c). These tasks were intended to replicate Gibson and Kingstone's (2006) study that had reported a cue type effect when observers' performance was compared across cue type conditions (see also Logan, 1995). Moreover, Gibson and Kingstone reported a cued axis effect when spatial word cues were shown, but not when arrow cues were shown. The tasks in Block ${ }_{\mathrm{w}}$ and Block were also intended to replicate Gibson et al.'s (2009) study, which reported a cued endpoint effect when spatial word cues were shown (and, more specifically, when the spatial word cue was either "Left" or "Right"), but not when arrow cues were shown. 


\section{Counterbalancing of experimental blocks with spatial cues}

If the ordering of experimental blocks in the present study were fixed, it is possible that the ordering of $\mathrm{Block}_{\mathrm{w}}$ and Block $_{\mathrm{a}}$ could bias the measurement of the cue type effect, in that the RTs for whichever block was presented second could be deflated by practice effects due to prior experience with the task during whichever block was presented first. Consequently, Block $\mathrm{w}_{\mathrm{w}}$ and Block $\mathrm{a}_{\mathrm{a}}$ were counterbalanced across two separate HITs in the present study. In other words, half of the workers $(n=15)$ completed one counterbalanced order of blocks (Block $\mathrm{nc}_{\mathrm{nc}}$, Block $\mathrm{w}_{\mathrm{w}}$, Block $\mathrm{a}_{\mathrm{a}}$ ), and the remaining half of workers $(n=15)$ completed a different counterbalanced order of blocks (Block $\mathrm{nc}_{\mathrm{nc}}$, Block $\mathrm{a}$, Block $_{\mathrm{w}}$ ). Given that the blocks differed in terms of the target context (unambiguous vs. ambiguous), the nature of the to-bediscriminated target stimuli ( $H / U$ letters vs. red/green circles), and the inclusion or omission of spatial cues (no cues vs. spatial word cues and arrow cues), there was no logical reason to anticipate carryover or practice effects from Block $\mathrm{nc}_{\mathrm{nc}}$ to Block $_{\mathrm{w}}$ or Block mize the number of HITs that would need to be posted for the purposes of counterbalancing, all workers completed Block $\mathrm{nc}_{\mathrm{nc}}$ first.

\section{Statistical power analyses}

Broadly, the planned statistical analyses in the present study can be categorized into two sets, on the basis of which effects of interest they were intended to test and which experimental block they were conducted in. The first set of analyses intended to test the leftright advantage was only conducted in Block $\mathrm{nc}_{\mathrm{nc}}$. However, the second set of analyses, intended to test the cue type, cued axis, and cued endpoint effects, was conducted in both Block $\mathrm{w}_{\mathrm{w}}$ and Block $\mathrm{a}$, and furthermore, one test was also planned with Experimental Block designated as an additional within-subjects factor. Accordingly, two a priori power analyses were conducted for the present study using the G*Power 3.1.9.2 computer program (Faul, Erdfelder, Lang, \& Buchner, 2007). All of the effects of interest in the present study were reported in the literature using different measures of effect size; nonetheless, all effect sizes were expected to be larger in magnitude than an analogous Cohen's $f$ of .30 (see Gibson \& Kingstone, 2006; Gibson et al., 2009; Pauszek \& Gibson, 2016). Thus, two separate power analyses were conducted in the present study to approximate the total sample size required to detect an effect size of Cohen's $f=.30$ or larger.

The first set of analyses, intended to test the left/right advantage, only involved a couple of paired-samples $t$ tests. Accordingly, the procedure was conducted with regard to a two-tailed, paired-samples $t$ test with the desired statistical power set at $(1-\beta)=.80$ and the alpha level set at $\alpha=.05$.
The results of this power analysis indicated that a total sample size greater than or equal to seven participants would be needed to detect the anticipated effect.

The second set of analyses, intended to test the cue type, cued axis, and cued endpoint effects, involved a series of paired-samples $t$ tests and a repeated measures analysis of variance (ANOVA), with Experimental Block designated as an additional within-subjects factor. Accordingly, the procedure was conducted with regard to the least powerful planned statistical test; that is, the three-way repeated measures ANOVA with Target Axis (vertical vs. horizontal), TargetDistractor Opposite Compatibility (compatible vs. incompatible), and Cue Type (spatial word cues vs. arrow cues) as the three within-subjects factors. Similar to the first set of analyses described above, the desired statistical power was set at $(1-\beta)$ $=.80$, and the alpha level was set at $\alpha=.05$. The results of this test indicated that a total sample size greater than or equal to 25 participants would be needed to detect the anticipated effects. Considering that this sample size was found to be larger than the sample size corresponding to the first power analysis, a total sample size of at least 25 workers was sought in the present study.

\section{Method}

\section{Participants}

Thirty unique workers were recruited through AMT to participate in the present study. This total sample size was slightly larger than the minimum sample size suggested by the pair of a priori power analyses because, admittedly, the expected quality of workers' data was underestimated before the HITs were posted. As a result, more workers were recruited than were sought after, with the expectation that some of the workers' data would need to be rejected for high error rates. A pair of post-hoc statistical power analyses revealed that the inclusion of the additional workers' data increased the estimated statistical power achieved in the two sets of analyses described above to $(1-\beta)>.99$ and $(1-\beta)=.89$, respectively. Accordingly, the present study is expected to have achieved sufficient statistical power to detect the anticipated effects.

Each worker accepted one of two HITs that were posted on AMT to allow for the counterbalancing of Block $\mathrm{w}_{\mathrm{w}}$ and Block $\mathrm{a}$. Each worker was compensated \$3.00 USD after completing the experiment, which took approximately 30 minutes to complete. To participate in the present study, each worker was required to (1) be a native and/or fluent English speaker, (2) self-report normal or corrected-to-normal visual acuity and normal color perception, (3) have a Windows-based operating system on their computer (to be compatible with webDMDX; see the Supplementary Materials), (4) finish the experiment with an overall percent error rate that was less than or equal 
to $20 \%$ (which was easy to do if they remained actively engaged in the task), and (5) completely read and agree with the terms outlined in the informed consent document provided on the experiment's HIT webpage.

In an effort to avoid deception and safeguard that all workers were indeed native and/or fluent English speakers, the Internet Protocol (IP) addresses of all workers participating in the present study were limited to those located in the United States. These IP addresses were also monitored to ensure that no single worker completed the same experimental condition more than once, or both conditions of the same experiment. Furthermore, in accordance with the recommendations of previous AMT replication studies (see, e.g., Enochson \& Culbertson, 2015), all workers in the present study were required to have a Master's qualification. The Master's qualification is a performance-based distinction awarded by AMT to elite groups of workers who have demonstrated a high degree of accuracy on specific types of HITs posted from a variety of different requesters. All workers with a Master's qualification must satisfy an ongoing battery of statistical monitoring to ensure that they continue to offer high-quality data that are deemed acceptable by the requesters. Any and all other demographic and personal information was not collected for the present study, and the identities of the workers remained completely anonymous.

\section{Ethics statement}

This research was conducted with the approval of the University of Notre Dame's Institutional Review Board (IRB). Prior to accepting the HIT, all workers were provided with the informed consent document and an overview of task instructions stating that clicking on the Link to Experiment button indicated voluntary participation, in addition to a complete understanding and acceptance of the terms outlined in the informed consent document. Written consent was waived by the University of Notre Dame's IRB, on the grounds that the research presented no more than minimal risk to participants and the study involved no procedures for which written consent is normally required outside of the research context.

\section{Apparatus, stimuli, and design}

A Web-deployable implementation of DMDX display software (Forster \& Forster, 2003) was used for all stimulus presentation and data collection. Typically, the DMDX display software requires researchers to undertake fine calibrations with their laboratory computers prior to testing, in order to control for variables such as the resolution and refresh rate of the computer monitor used for stimulus presentation. However, Forster and Forster's (2003) webDMDX software now enables Automode, wherein the resolutions and refresh rates of workers' computer monitors are automatically detected and accounted for during remote testing. Recently, Witzel et al. (2013) investigated whether the results of classic time-critical studies differed between the original, finecalibrated in-laboratory DMDX software and webDMDX's Automode for remote testing. In general, they found good consistency across platforms and concluded that webDMDX is a viable option for online experiments requiring fine temporal control of stimulus presentation and response recordings (Witzel et al., 2013).

Each experimental trial in the present study consisted of two displays presented on a black background: a fixation display and a (cue and) target display. In all three experimental blocks, the fixation display consisted of a small white dot $(0.35 \mathrm{~cm}$ in diameter $)$ presented in the center of the screen. However, the (cue and) target displays varied across experimental blocks, as will be described below in more detail.

In Block $\mathrm{nc}_{\mathrm{nc}}$, the target display did not include a cue; instead, the fixation dot remained in the center of the screen. The target displays also contained four letters, presented in white, that were located approximately $4.37 \mathrm{~cm}$ in each of the four cardinal directions away from fixation. Each letter was $1.43 \mathrm{~cm}$ tall and $0.84 \mathrm{~cm}$ wide. The target displays were unambiguous, in that they each contained only one target letter $(H$ or $U)$ and three distractors, which were randomly chosen without replacement from among the following seven letters: $S, P, L$, $C, E, A$, and $F$. Unambiguous target displays were used so that the workers did not require the aid of a spatial cue to correctly respond with the identity of the target letter. Accordingly, spatial cues were omitted from this block to avoid potential confounds associated with cue processing.

In Block $\mathrm{w}_{\mathrm{w}}$ and Block $\mathrm{a}$, a spatial cue replaced the fixation dot in the center of the screen when the cue and target displays appeared. In Block $\mathrm{w}_{\mathrm{w}}$, the cue was a spatial word cue ("ABOVE," "BELOW," "LEFT," or "RIGHT") presented in white and written in all capital letters. The spatial word cues were $0.71 \mathrm{~cm}$ tall and varied in width from 1.25 to $1.97 \mathrm{~cm}$. In Block $_{\mathrm{a}}$, the cue was an arrow cue $(\uparrow, \downarrow, \leftarrow$, or $\rightarrow)$ presented in white. The arrow cues varied in height and width from $0.49 \mathrm{~cm}$ to $1.16 \mathrm{~cm}$, depending on which direction they were pointing. The cue and target displays in both Block $\mathrm{w}_{\mathrm{w}}$ and Block $_{\mathrm{a}}$ also contained four colored target circles (each $1.27 \mathrm{~cm}$ in diameter) that were located approximately $4.37 \mathrm{~cm}$ in each of the four cardinal directions away from fixation. The target displays were ambiguous, in that two circles in each display were randomly selected to be colored red, and the remaining two circles were colored green. Ambiguous target displays were used to ensure that observers processed and used the different spatial cues fully and equally to complete the task. Otherwise, they would not be able to correctly respond with the color of the cued target (i.e., without guessing). 


\section{Procedure}

Typical display sequences in Block $\mathrm{nc}_{\mathrm{nc}}$, Block $\mathrm{w}_{\mathrm{w}}$, and Block $\mathrm{a}_{\mathrm{a}}$ are depicted in Fig. 1a, b, and c, respectively. Every trial in each block began with a fixation display presented for $500 \mathrm{~ms}$, followed immediately by the (cue and) target display. The (cue and) target display remained on the screen until a response was made (or until 4,000 ms elapsed). In Block $\mathrm{nc}_{\mathrm{nc}}$, workers' task was to locate and discriminate the identity ( $H$ or $U$ ) of the target letter present in each target display. In Block $_{\mathrm{w}}$ and Block $\mathrm{a}_{\mathrm{a}}$, workers' task was to locate and discriminate the color (red or green) of the target circle specified by the corresponding spatial cue for that block. In all three blocks, the to-be-discriminated target was equally likely to be presented at any one of the four potential target locations; moreover, the to-be-discriminated target was equally likely to be either an $H$ or a $U$ (in Block $\mathrm{nc}_{\text {c }}$ ) or either red or green (in Block $_{\mathrm{w}}$ and Block $\mathrm{a}_{\mathrm{a}}$. The spatial cues shown in Block $\mathrm{w}_{\mathrm{w}}$ and Block $_{\mathrm{a}}$ referred to each potential target location equally often.

Each block consisted of 100 trials preceded by comprehensive task instructions (including the illustrations depicted in Fig. 1a, b, and c) and ten practice trials. Workers were instructed to position their hands on their keyboard such that their left and right index fingers were resting on the $d$ and $j$ keys, respectively, throughout the experiment. In Block $\mathrm{nc}_{\mathrm{nc}}$, workers used their left hand to respond " $\mathrm{H}$ " and their right hand to respond "U." In Block $\mathrm{w}_{\mathrm{w}}$ and Block $\mathrm{a}_{\mathrm{a}}$, workers used their left hand to respond "red" and their right hand to respond "green." Note that Gibson and Kingstone (2005) investigated potential interactions between the response pad orientation (i.e., response keys located on the right or left of the response pad vs. on the top or bottom of the response pad) and cued axis, but found evidence for neither facilitation nor interference when, for example, observers had to orient in the left cued direction and respond using the left or right response key, respectively, relative to using the top or bottom response key (see also Gibson \& Davis, 2011).

\section{Results and discussion}

The mean correct RTs and percent error rates within each experimental block are listed in Table 1 as a function of experimental block, target axis, and target-distractor opposite compatibility (where applicable). Note that, in the present study, mean correct RTs greater than or equal to 3.0 standard deviations from the overall mean of each participant were trimmed to that value. Approximately $4.85 \%$ of the RTs in the present study were trimmed in this fashion. The effect sizes for any and all $t$ tests were estimated using Cohen's $d$, in accordance with the statistical procedures recommended by Cumming (2012).
In an effort to verify that the effects observed in the present study did not depend on the counterbalanced order of Block and Block $k_{\mathrm{a}}$, a four-way mixed ANOVA was conducted with Target Axis (vertical vs. horizontal), Target-Distractor Opposite Compatibility (compatible vs. incompatible), and Cue Type (spatial word cues vs. arrow cues) as the three withinsubjects factors, and with Counterbalanced Block Order $\left(\right.$ Block $_{\mathrm{w}}$, Block $_{\mathrm{a}}$ vs. Block $\mathrm{a}$, Block $\mathrm{w}_{\mathrm{w}}$ ) as the sole betweensubjects factor. Importantly, no main effects or interactions including the counterbalanced order of Block $\mathrm{w}_{\mathrm{w}}$ and Block approached statistical significance (all $p \mathrm{~s}>.285$ ). Thus, the order of counterbalanced blocks was confidently omitted as a between-subjects factor in all subsequent statistical analyses.

An independent set of statistical analyses was conducted within each experimental block of the present study in an effort to investigate the four attention effects of interest. Within each set of statistical analyses, the family-wise alphalevel was set at the conventionally accepted value of $\alpha=.05$. If multiple tests were conducted within the same set of statistical analyses, an additional Bonferroni correction was applied to account for repeated testing. The precise adjustment applied will be reported in each subsequent section, where applicable.

\section{The leftright advantage}

To investigate the expected left/right advantage in $\mathrm{Block}_{\mathrm{nc}}$, a couple of paired-samples $t$ tests were conducted comparing the mean correct RTs and percent error rates in response to targets presented on the vertical and horizontal axes. Note that these effects are thought to reflect target processing at isoeccentric locations in the absence of any spatial cuing. To account for repeated testing, these analyses were conducted using Bonferroni-adjusted family-wise alpha levels of $\alpha=$ $(.05 / 2)=.025$ per test.

The results indicated that RTs were significantly faster when the target appeared on the horizontal axis $(M=602 \mathrm{~ms})$ than when the target appeared on the vertical axis $(M=659 \mathrm{~ms})$, $t(29)=4.34, p<.001, d=0.36$. Despite this significant difference in RTs, the results indicated no significant difference in percent error rates when the target appeared on the horizontal axis $(M=3.69)$, relative to when the target appeared on the vertical axis $(M=2.65), t(29)=1.02, p=.317, d=0.26$. Thus, we found no evidence suggesting that a speed-accuracy tradeoff was associated with target processing in Block $\mathrm{nc}_{\mathrm{nc}}$. Taken together, these results successfully replicated the left/right advantage reported in previous studies of the symbolic control of attention (see, e.g., Pauszek \& Gibson, 2016).

\section{The cue type effect}

To investigate the expected cue type effect between Block $\mathrm{w}_{\mathrm{w}}$ and Block , a couple of paired-samples $t$ tests were conducted comparing the overall mean correct RTs and percent error 
Table 1 Mean correct RTs (in milliseconds) and error rates (percentages) for the present study, listed as a function of experimental block, target axis, and target-distractor opposite compatibility (where applicable)

\begin{tabular}{|c|c|c|c|c|c|c|}
\hline \multirow[t]{2}{*}{ Target Axis } & \multicolumn{2}{|l|}{ Block $_{\mathrm{nc}}$} & \multicolumn{2}{|l|}{ Block $_{\mathrm{w}}$} & \multicolumn{2}{|l|}{ Block $_{\mathrm{a}}$} \\
\hline & $\mathrm{RT}(S E)$ & Errors $(S E)$ & $\mathrm{RT}(S E)$ & Errors $(S E)$ & $\mathrm{RT}(S E)$ & Errors $(S E)$ \\
\hline \multicolumn{7}{|l|}{ Vertical } \\
\hline Overall & $659(32.35)$ & $2.65(0.79)$ & $911(34.08)$ & $2.51(0.61)$ & $631(23.78)$ & $2.81(0.58)$ \\
\hline Opposite-compatible & N/A & N/A & $913(34.87)$ & $2.95(0.94)$ & $634(26.95)$ & $2.33(0.71)$ \\
\hline Opposite-incompatible & N/A & N/A & $908(34.86)$ & $2.06(0.40)$ & $629(21.61)$ & $3.28(0.79)$ \\
\hline \multicolumn{7}{|l|}{ Horizontal } \\
\hline Overall & $602(24.59)$ & $3.69(0.66)$ & $1,069(54.15)$ & $7.94(1.12)$ & $632(25.43)$ & $2.65(0.50)$ \\
\hline Opposite-compatible & $\mathrm{N} / \mathrm{A}$ & N/A & $1,016(52.18)$ & $4.41(0.91)$ & $634(25.42)$ & $2.70(0.78)$ \\
\hline Opposite-incompatible & N/A & N/A & $1,122(57.94)$ & $11.46(1.62)$ & $631(26.55)$ & $2.59(0.55)$ \\
\hline
\end{tabular}

Standard errors are shown in parentheses

rates in these blocks. To account for repeated testing, these analyses were conducted using Bonferroni-adjusted familywise alpha levels of $\alpha=(.05 / 2)=.025$ per test.

The results indicated that the overall RTs were significantly faster when arrow cues were used in $\operatorname{Block}_{\mathrm{a}}(M=632 \mathrm{~ms})$ than when spatial word cues were used in $\operatorname{Block}_{\mathrm{w}}(M=990 \mathrm{~ms})$, $t(29)=14.55, p<.001, d=1.88$. In addition, the results indicated that the overall percent error rates were significantly higher when spatial word cues were used in $\operatorname{Block}_{\mathrm{w}}(M=$ 5.22) than when arrow cues were used in $\operatorname{Block}_{\mathrm{a}}(M=2.73)$, $t(29)=4.05, p<.001, d=0.70$. Taken together, these results successfully replicated the cue type effect reported in previous studies comparing observers' performance using spatial word cues and arrow cues (see, e.g., Gibson \& Kingstone, 2006; Gibson et al., 2009; Logan, 1995).

\section{The cued axis effect}

To investigate the expected presence and absence of the cued axis effect in Block $\mathrm{w}_{\mathrm{w}}$ and Block $\mathrm{a}$, respectively, a series of paired-samples $t$ tests were conducted comparing the mean correct RTs and percent error rates in response to targets presented on the vertical and horizontal axes. To account for repeated testing, these analyses were conducted using Bonferroni-adjusted family-wise alpha levels of $\alpha=(.05 / 4)$ $=.0125$ per test.

In response to the spatial word cues used in $\mathrm{Block}_{\mathrm{w}}$, the results indicated that RTs were significantly faster when the target appeared on the vertical axis $(M=911 \mathrm{~ms})$, relative to when the target appeared on the horizontal axis $(M=1,069$ $\mathrm{ms}), t(29)=5.46, p<.001, d=0.64$. In addition, the results indicated that the percent error rates were significantly higher when the target appeared on the horizontal axis $(M=7.94)$ than when the target appeared on the vertical axis $(M=2.51)$, $t(29)=6.66, p<.001, d=1.10$. Taken together, these results successfully replicated the cued axis effect reported in previous studies using spatial word cues (see, e.g., Gibson \& Kingstone, 2006; Gibson et al., 2009; Logan, 1995).

In response to the arrow cues used in Block ${ }_{\mathrm{a}}$, the results indicated no significant difference in RTs when the target appeared on the vertical axis $(M=631 \mathrm{~ms})$, relative to when the target appeared on the horizontal axis $(M=632 \mathrm{~ms}), t(29)=$ $0.13, p=.901, d=0.01$. In addition, the results also indicated no significant difference in percent error rates when the target appeared on the vertical axis $(M=2.81)$, relative to when the target appeared on the horizontal axis $(M=2.65), t(29)=0.24$, $p=.810, d=0.05$. Taken together, these results successfully replicated the null effects of target axis reported in previous studies using arrow cues (see, e.g., Gibson \& Kingstone, 2006; Gibson et al., 2009; Logan, 1995).

\section{The cued endpoint effect}

To investigate the expected presence and absence of the cued endpoint effect in Block $\mathrm{w}_{\mathrm{w}}$ and Block $\mathrm{a}_{\mathrm{a}}$, respectively, a three-way repeated measures ANOVA was conducted on the mean correct RTs and percent error rates, with Target Axis (vertical vs. horizontal), Target-Distractor Opposite Compatibility (compatible vs. incompatible), and Cue Type (spatial word cues vs. arrow cues) as the three within-subjects factors. Critically, the results of this test indicated that all main effects and lower-order interactions of RTs were qualified by a significant three-way interaction between target axis, targetdistractor opposite compatibility, and cue type, $F(1,29)=$ $10.50, p=.003, \eta_{\mathrm{p}}^{2}=.27$. This three-way interaction was also found to be significant with respect to the percent error rates, $F(1$, 29) $=15.54, p<.001, \eta_{\mathrm{p}}{ }^{2}=.35$. Accordingly, these significant three-way interactions were investigated further by conducting two-way repeated measures ANOVAs within each Cue Type condition (spatial word cues vs. arrow cues) separately, with Target Axis (vertical vs. horizontal) and Target-Distractor Opposite Compatibility (compatible vs. incompatible) as the 
two within-subjects factors. To account for repeated testing, these analyses were conducted using Bonferroni-adjusted family-wise alpha levels of $\alpha=(.05 / 4)=.0125$ per test.

In terms of RTs in response to the spatial word cues used in Block $_{\mathrm{w}}$, the results of the two-way repeated measures ANOVA indicated a significant two-way interaction between target axis and target-distractor opposite compatibility, $F(1$, $29)=20.95, p<.001, \eta_{\mathrm{p}}{ }^{2}=.42$. Subsequent paired-samples $t$ tests were conducted to compare the effects of targetdistractor opposite compatibility within each target axis condition. The results indicated no significant difference in RTs when the target appeared on the vertical axis and the target and opposite distractor colors were either compatible $(M=913$ $\mathrm{ms}$ ) or incompatible ( $M=908 \mathrm{~ms}), t(29)=0.40, p=.696, d$ $=0.03$. In contrast, the results also indicated that RTs were significantly faster when the target appeared on the horizontal axis and the target and opposite distractor colors were compatible $(M=1,016 \mathrm{~ms})$, relative to when they were incompatible $(M=1,122 \mathrm{~ms}), t(29)=5.12, p<.001, d=0.35$.

In terms of percent error rates in response to the spatial word cues used in Block $\mathrm{w}_{\mathrm{w}}$, the results of the two-way repeated measures ANOVA indicated a significant two-way interaction between target axis and target-distractor opposite compatibility, $F(1,29)=17.46, p<.001, \eta_{\mathrm{p}}{ }^{2}=.38$. Subsequent pairedsamples $t$ tests were conducted to compare the effects of target-distractor opposite compatibility within each target axis condition. The results indicated no significant difference in percent error rates when the target appeared on the vertical axis and the target and opposite distractor colors were either compatible $(M=2.95)$ or incompatible $(M=2.06), t(29)=$ $1.15, p=.259, d=0.23$. In contrast, the results also indicated that percent error rates were significantly higher when the target appeared on the horizontal axis and the target and opposite distractor colors were incompatible $(M=11.46)$, relative to when they were compatible $(M=4.41), t(29)=5.17, p$ $<.001, d=0.98$. Taken together, these results successfully replicated the cued endpoint effect reported in previous studies using spatial word cues (see, e.g., Gibson et al., 2009).

Identical two-way repeated measures ANOVAs were conducted on the mean correct RTs and percent error rates in response to the arrow cues used in Block ${ }_{\mathrm{a}}$. However, no main effects or interactions approached statistical significance with respect to the RTs (all $p \mathrm{~s}>.570$ ), nor with respect to the percent error rates (all $p \mathrm{~s}>.450$ ). Taken together, these results successfully replicated the null effects of target-distractor opposite compatibility reported in previous studies using arrow cues (see, e.g., Gibson et al., 2009).

\section{General discussion}

The present study was conducted in an effort to validate AMT as a viable option for remotely conducting visual attention experiments using the spatial cuing paradigm, which requires fine temporal control of stimulus presentation and response recordings. Four hallmark attention effects reported in previous studies of spatial cuing and the semantics of space were successfully replicated using a Web-deployable implementation of the DMDX display software (Forster \& Forster, 2003). In general, these findings provide support for the conclusions of Witzel et al. (2013), in that there appears to be good consistency between the traditional DMDX platform, intended for use in a laboratory setting, and the webDMDX platform with Automode, intended for use in remote testing. All effects of interest in the present study were found to successfully replicate, and therefore it seems unlikely that the origins of these effects lie in some extraneous factors involving the fine calibrations of laboratory computers, the controlled laboratory setting itself, or the in-person task instructions given to participants. Moreover, the detection of these effects does not appear to be mired by the myriad of unpredictable factors associated with remotely collecting data online. These conclusions are both theoretically and practically important, because the present study provides sufficient evidence to conclude that AMT is a viable method for efficiently and reliably investigating the symbolic control of attention and, more generally, visual attention as a whole.

\section{Comparing AMT data with laboratory data}

A major concern associated with collecting behavioral data over the Internet is the potential for increased variability resulting from a more diverse population of participants or unknown differences in the fine calibrations (e.g., resolutions and refresh rates) of workers' personal computers used for testing. Although these potential issues did not appear to influence the qualitative findings of the present AMT data (i.e., all of the effects of interest from previously published laboratory studies were successfully replicated), it is possible that the present AMT data might be quantitatively different from the data collected in analogous laboratory experiments. Thus, the present AMT data were directly compared to the corresponding previously published laboratory data, as we describe below in more detail.

The mean correct RT and error rate effect sizes derived from the present AMT study and from previously published laboratory studies are listed for each effect of interest in Table 2. Note that all the effect sizes listed in Table 2 were calculated or recalculated in the same manner, in an effort to retain consistency across effect size computations. The sample sizes, overall error rates, and descriptive statistics for the present AMT study and the corresponding laboratory studies are also listed in Tables 3 (for the left/right advantage), 4 (for the cue type and cued axis effects), and 5 (for the cued endpoint effect). Subsequent statistical analyses were conducted in an effort to ascertain whether the present AMT data 
Table 2 Mean correct RT and error rate effect sizes derived from the present AMT study and from previously published laboratory studies

Effect of Interest

\begin{tabular}{|c|c|c|c|c|}
\hline & Left/Right Advantage & Cue Type Effect & Cued Axis Effect & Cued Endpoint Effect \\
\hline & \multicolumn{4}{|l|}{ Mean Correct RTs } \\
\hline \multicolumn{5}{|l|}{ Effect size } \\
\hline AMT study & Cohen's $d=0.36$ & Cohen's $d=1.88$ & Cohen's $d=0.64$ & $\eta_{\mathrm{p}}^{2}=.42$ \\
\hline \multirow[t]{2}{*}{ Laboratory study } & Cohen's $d=0.36^{\mathrm{a}}$ & Cohen's $d=1.91^{\mathrm{b}}$ & Cohen's $d=0.28^{\mathrm{b}}$ & $\eta_{\mathrm{p}}^{2}=.36^{\mathrm{c}}$ \\
\hline & Percent Error Rates & & & \\
\hline \multicolumn{5}{|l|}{ Effect size } \\
\hline AMT study & Cohen's $d=0.26$ & Cohen's $d=0.70$ & Cohen's $d=1.10$ & $\eta_{\mathrm{p}}^{2}=.38$ \\
\hline Laboratory study & Cohen's $d=0.12^{\mathrm{a}}$ & Cohen's $d=0.34^{\mathrm{b}}$ & Cohen's $d=0.13^{\mathrm{b}}$ & $\eta_{\mathrm{p}}^{2}<.01^{\mathrm{c}}$ \\
\hline
\end{tabular}

Only effect sizes from the primary, statistically significant main effect or interaction for each effect of interest (see the Results and discussion section) are included. Where necessary, the laboratory data were reanalyzed in an effort to directly mirror the statistical analyses conducted in the present AMT study. ${ }^{a}$ Data from Pauszek and Gibson (2016, Exp. 1). ${ }^{\mathrm{b}}$ Data from the 0-ms SOA condition within the spatial word and arrow cue type conditions of Gibson and Kingstone (2006). ${ }^{\mathrm{c}}$ Data from the 0-ms SOA condition of Gibson, Scheutz, and Davis (2009, Exp. 1)

quantitatively differed from the laboratory data obtained in previously published studies.

With respect to mean correct RTs, a series of independentsamples $t$ tests failed to reveal any statistically significant differences between the present AMT data and the corresponding laboratory data (all $p \mathrm{~s}>.280$ ). However, a separate series of independent-samples $t$ tests revealed an interesting, albeit unexpected, pattern of results with respect to the overall percent error rates. Specifically, the analyses revealed that error rates in the spatial word cue and arrow cue conditions of Gibson and Kingstone's (2006) study $(M=7.17)$ were significantly higher than those in Block $\mathrm{w}_{\mathrm{w}}$ and Block $\mathrm{a}_{\mathrm{a}}$ of the present AMT study $(M=3.97), t(44)=2.48, p=.017, d=0.70$. The analyses also revealed that error rates in the 0 -ms stimulus-onset asynchrony (SOA) condition of Gibson et al.'s (2009) Experiment $1(M=8.91)$ were significantly higher than those in Block $_{\mathrm{w}}$ of the present AMT study $(M=5.22), t(64)=2.70, p=.009, d=$

Table 3 Mean correct RTs (in milliseconds) and error rates (percentages), listed as a function of target axis in the present AMT study and in a previously published study aimed at investigating the leftright advantage

\begin{tabular}{|c|c|c|c|c|}
\hline & \multicolumn{2}{|l|}{ Dataset } & & \\
\hline & \multicolumn{2}{|c|}{ AMT Study ${ }^{\mathrm{a}}$} & \multicolumn{2}{|c|}{ Laboratory Study ${ }^{\mathrm{b}}$} \\
\hline & $\mathrm{RT}(S D)$ & Errors $(S D)$ & $\mathrm{RT}(S D)$ & Errors $(S D)$ \\
\hline Sample size & \multicolumn{2}{|l|}{$N=30$} & \multicolumn{2}{|l|}{$N=20$} \\
\hline Overall error rates & \multicolumn{2}{|c|}{$M=3.17 \%, S D=2.84 \%$} & \multicolumn{2}{|c|}{$M=3.54 \%, S D=2.16 \%$} \\
\hline \multicolumn{5}{|c|}{ Target axis } \\
\hline Vertical & $659(177)$ & $2.65(4.35)$ & $632(140)$ & $3.68(2.45)$ \\
\hline Horizontal & $602(135)$ & $3.69(3.61)$ & $584(129)$ & $3.40(2.09)$ \\
\hline
\end{tabular}

The sample size and overall error rates for each study are also included. Standard deviations are shown in parentheses. ${ }^{\text {a }}$ Data from Block $_{\mathrm{nc}}$ of the present study. ${ }^{b}$ Data from Pauszek and Gibson (2016, Exp. 1)
0.68. It is interesting to note that the error rates in Pauszek and Gibson's (2016) study ( $M=3.54)$ were also numerically higher than those in Block ${ }_{\mathrm{nc}}$ of the present AMT study $(M=$ 3.17), although this difference was not found to be statistically significant, $t(48)=0.49, p=.624, d=0.15$.

Enochson and Culbertson (2015) recently reported a similar unexpected pattern of results in their study investigating the online replicability of certain psycholinguistic effects reported in previous laboratory studies. In particular, the authors reported that both the overall mean RTs and overall standard error rates captured in their laboratory data were significantly larger than those captured using AMT. According to the authors, the finding of faster mean RTs obtained from AMT workers is not surprising, when considering that they are essentially paid by the hour. Thus, AMT workers are incentivized to work as quickly as possible on each task and to complete as many tasks as they can over a given period of time. This finding may not have been observed in the present study, due to the temporal structure of the present experiments, relative to the experiments employed by Enochson and Culbertson (2015). In other words, the participants in the present experiments (both AMT-based and laboratory-based) were provided with a 4,000-ms window during which they could respond on each trial. As a result, the overall distribution of mean correct RTs in these tasks was somewhat restricted. In contrast, Enochson and Culbertson's (2015) self-paced reading tasks allowed for a wider distribution of overall RTs that were primarily dependent on the natural reading pace of each participant. Thus, it is reasonable to conclude that any differences in mean correct RTs observed between AMT-based and laboratory-based experiments varies on an experiment-toexperiment basis and relies on a variety of factors, such as the structure and temporal constraints of the experiment itself.

Enochson and Culbertson (2015) also reported significantly larger standard error values and (in their case, anecdotally) 
Table 4 Mean correct RTs (in milliseconds) and error rates (percentages), listed as a function of cue type and target axis in the present AMT study and in a previously published study aimed at investigating the cue type and cued axis effects

\begin{tabular}{|c|c|c|c|c|}
\hline & \multicolumn{2}{|l|}{ Dataset } & & \\
\hline & \multicolumn{2}{|c|}{ AMT Study ${ }^{\mathrm{a}}$} & \multicolumn{2}{|c|}{ Laboratory Study ${ }^{\mathrm{b}}$} \\
\hline & $\mathrm{RT}(S D)$ & Errors $(S D)$ & $\mathrm{RT}(S D)$ & Errors $(S D)$ \\
\hline Sample size & \multicolumn{2}{|l|}{$N=30$} & \multicolumn{2}{|l|}{$N=16$} \\
\hline Overall error rates & \multicolumn{2}{|c|}{$M=3.97 \%, S D=3.12 \%$} & \multicolumn{2}{|c|}{$M=7.17 \%, S D=5.65 \%$} \\
\hline \multicolumn{5}{|l|}{ Cue type } \\
\hline Spatial word cues & $990(235)$ & $5.22(4.41)$ & $1,007(212)$ & $8.72(7.80)$ \\
\hline Arrow cues & $632(133)$ & $2.73(2.39)$ & $597(88)$ & $5.61(4.48)$ \\
\hline \multicolumn{5}{|l|}{ Target axis ${ }^{c}$} \\
\hline Vertical & $911(187)$ & $2.51(3.34)$ & $976(214)$ & $8.14(9.75)$ \\
\hline Horizontal & $1,069(297)$ & $7.94(6.14)$ & $1,037(224)$ & $9.29(7.11)$ \\
\hline
\end{tabular}

The sample size and overall error rates for each study are also included. Standard deviations are shown in parentheses. ${ }^{\mathrm{a}}$ Data from Block ${ }_{\mathrm{w}}$ and Block $\mathrm{a}_{\mathrm{a}}$ of the present study. ${ }^{\mathrm{b}}$ Data from the 0 -ms SOA condition within the spatial word and arrow cue type conditions of Gibson and Kingstone (2006). ${ }^{\mathrm{c}}$ Data from Block $\mathrm{w}_{\mathrm{w}}$ of the present study and the 0 -ms SOA condition within the spatial word cue condition of Gibson and Kingstone (2006)

higher error rates when the data were collected in the laboratory, relative to when they were collected online via AMT. This finding certainly seems counterintuitive at first glance. However, in hindsight, there are a number of reasonable explanations for this effect. For one, in the same vein as the discussion above, it is possible that AMT workers may be more compliant with task instructions, due to the fact that they are directly compensated for their participation with monetary payment. Undergraduate participants in the laboratory, on the other hand, are typically compensated for their participation with partial course credit. Another potential explanation for this finding could be that AMT workers in the present study were required to achieve an overall error rate that was less than or equal to $20 \%$ in order to receive compensation. Participants in laboratory studies, on the other hand, do not typically have any such qualification; rather, they receive partial course credit for their participation regardless of the quality of their data. It is therefore possible that the AMT workers were more highly motivated than participants in the laboratory, due to both the nature of their compensation and the fact that they were required to perform adequately on the task to be compensated.

Table 5 Mean correct RTs (in milliseconds) and error rates (percentages), listed as a function of target axis and target-distractor opposite compatibility in the present AMT study and in a previously published study aimed at investigating the cued endpoint effect

\begin{tabular}{|c|c|c|c|c|}
\hline & \multirow{2}{*}{\multicolumn{2}{|c|}{$\begin{array}{l}\text { Dataset } \\
\text { AMT Study }^{\mathrm{a}}\end{array}$}} & & \\
\hline & & & \multicolumn{2}{|c|}{ Laboratory Study ${ }^{\mathrm{b}}$} \\
\hline & $\mathrm{RT}(S D)$ & Errors $(S D)$ & $\mathrm{RT}(S D)$ & Errors $(S D)$ \\
\hline Sample size & \multicolumn{2}{|l|}{$N=30$} & \multicolumn{2}{|l|}{$N=36$} \\
\hline Overall error rates & \multicolumn{2}{|c|}{$M=5.22 \%, S D=4.41 \%$} & \multicolumn{2}{|c|}{$M=8.91 \%, S D=6.30 \%$} \\
\hline \multicolumn{5}{|l|}{ Target axis } \\
\hline \multicolumn{5}{|l|}{ Vertical } \\
\hline Overall & 911 (187) & $2.51(3.34)$ & $926(184)$ & $7.75(7.05)$ \\
\hline Opposite-compatible & $913(191)$ & $2.95(5.15)$ & 937 (197) & $6.99(8.43)$ \\
\hline Opposite-incompatible & $908(191)$ & $2.06(2.21)$ & $915(178)$ & $8.51(7.21)$ \\
\hline \multicolumn{5}{|l|}{ Horizontal } \\
\hline Overall & $1,069(297)$ & $7.94(6.14)$ & $1,010(263)$ & $10.07(6.85)$ \\
\hline Opposite-compatible & $1,016(286)$ & $4.41(4.98)$ & $975(241)$ & $8.96(8.33)$ \\
\hline Opposite-incompatible & $1,122(317)$ & $11.46(8.86)$ & $1,045(293)$ & $11.18(7.38)$ \\
\hline
\end{tabular}

The sample size and overall error rates for each study are also included. Standard deviations are shown in parentheses. ${ }^{\mathrm{a}}$ Data from Block $\mathrm{w}_{\mathrm{w}}$ of the present study. ${ }^{\mathrm{b}}$ Data from the 0-ms SOA condition of Gibson, Scheutz, and Davis (2009, Exp. 1) 
In sum, the present AMT experiments were successful in qualitatively replicating the effects of interest in the present study. Furthermore, the present AMT data generally provided lower error rates than analogous studies conducted in the laboratory. Future endeavors should aim to reveal whether the latter unexpected finding indeed arises in AMT replication studies due to the abovementioned hypothetical differences in participant compensation and motivation. Such investigations could reveal a number of potentially important factors that elicit dissimilarities between AMT-based and laboratorybased data. As an added benefit, these studies may also provide a number of practical applications for researchers with respect to ways in which the participants in laboratory studies can be more highly incentivized to comply with task instructions and provide higher-quality data.

\section{Benefits and drawbacks of AMT}

The most obvious benefit of using AMT as a remote data collection method is the rate at which an adequate sample size can be attained. For example, a complete dataset from the entire population of workers who participated in the present study $(N=30)$ was collected in approximately 18 hours. This ability to rapidly collect adequate sample sizes is paramount to the appeal of AMT, since anecdotal evidence suggests that obtaining an equivalent sample size in the laboratory would likely have taken multiple weeks.

Another benefit of using AMT is the ability to exclusively study subsects of its large and diverse population of workers by specifying a variety of different worker qualifications. In the present study, spatial word cues written in English text were used and, accordingly, the pool of potential workers eligible for participation was restricted to those with IP addresses located in the United States. Nevertheless, researchers now have the ability to investigate the symbolic control of attention through AMT in populations with any number of different cultures and native languages. For most researchers, this ability has previously been unattainable, especially for those who collect data at smaller universities with limited populations of foreign students. Such endeavors have the potential to unveil novel insights into the nature of visual attention across humanity as a whole. It should be mentioned that, as of 2010 , an estimated $46.8 \%$ and $34.0 \%$ of the total workers the comprise AMT's workforce were reported to be residents of the United States and India, respectively (Ipeirotis, 2010). Therefore, future studies should examine whether these estimations have substantially changed in recent years; if not, they should also aim to further estimate the diversity (or lack thereof) of the remaining approximate $19.2 \%$ of AMT's reported workforce. Such investigations would undoubtedly be useful in shedding light on the extent to which obtaining a truly diverse sample is feasible when collecting data through AMT.
Despite their obvious value for accelerating the rate of data collection and potentially increasing the diversity of samples, online crowdsourcing services like AMT are not without their drawbacks. For instance, the testing environment surrounding the worker during testing cannot be controlled, or even known, when collecting data remotely. In the present study, we found no evidence suggesting that workers were distracted significantly more than in a controlled laboratory setting (i.e., the percent error rates in the present AMT study were actually lower than those in analogous studies conducted in the laboratory, as we will elaborate on in a subsequent section). However, the potential for external distraction to significantly influence workers' performance likely varies on a sample-to-sample and experiment-to-experiment basis. Moreover, online experiments that keep the participants' identities anonymous may attract scammers to utilize bots in an effort to receive compensation while subverting authentic participation. A final potential drawback of AMT is the existence of so-called Professional Turkers, or workers on AMT who actively seek out homologous psychological experiments and participate in a large number of similar studies. The extent to which each workers' performance on any given experiment may vary as a function of previous experiences with homologous experiments would be an interesting avenue for future research. Regardless, it is expected that this issue would be more problematic for some experimental paradigms than for others, on the basis of the extent to which the performance in an experiment is susceptible to bias from carryover or practice effects. Fortunately, the HIT interface and worker qualifications permitted by AMT allow for a number of these concerns to be alleviated, at least with some degree of confidence. Thus, to conclude, a number of recommendations for future endeavors using AMT are provided below in an effort to aid those interested in controlling for potential confounds when remotely collecting data online.

\section{Recommendations for future endeavors using AMT}

The present study suggests a number of recommendations for future endeavors using AMT as a means of collecting behavioral data online. These recommendations are outlined in the following sections. Additional recommendations that are specific to the use of external experimental software (e.g., webDMDX) are provided in the Supplementary Materials.

Counterbalancing experimental conditions As was previously described, in the present study we counterbalanced Block $_{\mathrm{w}}$ and Block $\mathrm{a}_{\mathrm{a}}$ in an effort to avoid any bias due to potential carryover or practice effects. If future studies require counterbalancing of within-subjects blocks, requesters will need to post multiple HITs, each containing a different ordering of blocks. In so doing, the requester would be well-served to monitor the IP addresses of all workers across these HITs, to ensure that no single worker completes the same experimental 
condition more than once, or both conditions of the same experiment. An instruction should also be included on each HIT webpage instructing workers that they will only be compensated once for their participation in the study, and that repeated participation will not be compensated. Alternatively, a number of services are available for free online that will automatically monitor individual workers' participation across multiple HITs; Unique Turker (https://uniqueturker.myleott.com) is one of the more popular aids that service this need. Fortunately, the instructions alone appeared to be sufficient for eliciting worker compliance in the present study, as no duplicate IP addressed were found to participate in the same HIT more than once, nor in both counterbalanced conditions. However, this finding may simply reflect the relatively small sample sizes that were obtained in the present study. That said, future studies could investigate the extent to which workers on AMT subvert instructions, either intentionally or accidentally, and participate in the same HIT more than once, or in more than one counterbalanced condition of the same experiment. It may also be useful to investigate the possibility that worker compliance in this regard varies on the basis of whether or not workers have achieved a Master's qualification.

Worker qualifications As was also previously described, the present study required all workers to have achieved an AMTdesignated Master's qualification prior to participating. Specifying this qualification ensures that only the highestquality workers are permitted to participate in experiments, although this benefit does come at a cost. Specifically, AMT charges requesters an additional 5\% fee on top of each workers' compensation. In the present study, the total cost of restricting the pool of potential workers to those with Master's qualifications was approximately $\$ 4.50$. Considering that every single worker provided acceptable data with relatively low (i.e., less than 20\%) error rates, it appears that this qualification is worth the additional cost. This notion was further supported by Enochson and Culbertson (2015), in a recent study investigating the online replicability of certain psycholinguistic effects observed in previous laboratory studies. In general, the authors anecdotally reported that a sample of workers without Master's qualifications yielded significantly higher error rates, and a higher overall data rejection rate, than did a sample of workers with Master's qualifications. Unfortunately, the authors did not perform any statistical analyses directly comparing task performance across samples of workers either with or without a Master's qualification. Therefore, future studies should also aim to unveil whether requiring workers to have achieved a Master's qualification indeed improves the quality of data by reducing overall error rates and further ensuring compliance with task instructions.

Establishing an error rate requirement As was also previously described, the present study required all workers to have achieved an overall percent error rate that was less than or equal to $20 \%$ in order to be compensated for their participation. This value was selected because, anecdotally, achieving this error rate is easy to do if the observer follows instructions and remains actively engaged in the task. Fortunately, all workers who participated in the present study were able to achieve an adequately low error rate, although this might also reflect the fact that all workers had a Master's qualification. Nevertheless, future studies should investigate whether minimal error rate requirements indeed influence workers' performance by reducing overall error rates and further ensuring compliance with task instructions.

\section{Conclusions}

In conclusion, the findings of the present study suggest that AMT is a viable option for remotely collecting data online to investigate the symbolic control of attention using the spatial cuing paradigm. Four hallmark effects previously observed in the laboratory were successfully replicated using AMT, despite the myriad of unpredictable factors associated with remote testing on workers' own computers. Consequently, the present study provides a crucial first step toward opening new avenues for efficiently and reliably investigating the symbolic control of attention in large and diverse samples that more accurately reflect the diversity of all humanity. Despite their drawbacks, online crowdsourcing services like AMT have the potential to completely revolutionize the way in which psychological research is typically conducted. The use of these services affords researchers the ability to rapidly recruit large and potentially diverse samples of workers, in addition to allowing for the rapid collection of pilot data that can be used to finalize experimental procedures and methodologies prior to the execution of largescale studies. Thus, in sum, AMT has the potential to evolve into a vital tool that can be used to alleviate a number of aggravations associated with collecting behavioral data. Researchers would be wise to further validate its use in their respective fields of study by replicating hallmark effects that have been previously reported in controlled laboratory experiments. In so doing, they afford themselves with the opportunity to accelerate not only their own scientific contributions, but also those of their colleagues.

\section{References}

Abrams, J., Nizam, A., \& Carrasco, M. (2012). Isoeccentric locations are not equivalent: The extent of the vertical meridian asymmetry. Vision Research, 52, 70-78. doi:10.1016/j.visres.2011.10.016

Bates, J. A., \& Lanza, B. A. (2013). Conducting psychology student research via the Mechanical Turk crowdsourcing service. North American Journal of Psychology, 15, 385-394. 
Becker, M., Nevins, A., \& Levine, J. (2012). Asymmetries in generalizing alternations to and from initial syllables. Language, 88, 231-268.

Buhrmester, M., Kwang, T., \& Gosling, S. D. (2011). Amazon's Mechanical Turk: A new source of inexpensive, yet high-quality, data? Perspectives on Psychological Science, 6, 3-5. doi:10.1177/1745691610393980

Carrasco, M. (2014). Spatial covert attention: Perceptual modulation. In A. C. Nobre \& S. Kastner (Eds.), The Oxford handbook of attention (pp. 183-230). New York, NY: Oxford University Press.

Connolly, M., \& Van Essen, D. C. (1984). The representation of the visual field in parvicellular and magnocellular layers of the lateral geniculate nucleus in the macaque monkey. Journal of Comparative Neurology, 226, 544-564.

Crump, M. J. C., McDonnell, J. V., \& Gureckis, T. M. (2013). Evaluating Amazon's Mechanical Turk as a tool for experimental behavioral research. PLoS ONE, 8(e57410), 1-18. doi:10.1371/journal. pone. 0057410

Cumming, G. (2012). Understanding the new statistics: Effect sizes, confidence intervals, and meta-analyses. New York, NY: Routledge.

Curcio, C. A., \& Allen, K. A. (1990). Topography of ganglion cells in human retina. The Journal of Comparative Neurology, 300, 5-25.

Curcio, C. A., Sloan, K. R., Packer, O., Hendrickson, A. E., \& Kalina, R. E. (1987). Distribution of cones in human and monkey retina: Individual variability and radial asymmetry. Science, 236, 579-582.

Curcio, C. A., Sloan, K. R., Kalina, R. E., \& Hendrickson, A. E. (1990). Human photoreceptor topography. The Journal of Comparative Neurology, 292, 497-523.

Enochson, K., \& Culbertson, J. (2015). Collecting psycholinguistic response time data using Amazon Mechanical Turk. PLoS ONE, 10(e116946), 1-17. doi:10.1371/journal.pone.0116946

Faul, F., Erdfelder, E., Lang, A.-G., \& Buchner, A. (2007). G*Power 3: A flexible statistical power analysis program for the social, behavioral, and biomedical sciences. Behavior Research Methods, 39, 175-191. doi:10.3758/BF03193146

Fine, A. B., Jaeger, T. F., Farmer, T. A., \& Qian, T. (2013). Rapid expectation adaptation during syntactic comprehension. PLOS ONE, 8(e77661), 1-18. doi:10.1371/journal.pone.0077661

Forster, K. I., \& Forster, J. C. (2003). DMDX: A Windows display program with millisecond accuracy. Behavior Research Methods, Instruments, \& Computers, 35, 116-124. doi:10.3758/BF03195503

Gibson, B. S., \& Davis, G. J. (2011). Grounding spatial language in the motor system: Reciprocal interactions between conceptual control and spatial orienting. Visual Cognition, 19, 79-116.

Gibson, B. S., \& Kingstone, A. F. (2005). Visual attention and the semantics of space: Beyond central and peripheral cues. Paper presented at the 46th Annual Meeting of the Psychonomic Society, Toronto, CA.

Gibson, B. S., \& Kingstone, A. (2006). Visual attention and the semantics of space: Beyond central and peripheral cues. Psychological Science, 17, 622-627.

Gibson, B. S., \& Sztybel, P. (2014). The spatial semantics of symbolic attention control. Current Directions in Psychological Science, 23, 271-276.

Gibson, B. S., Scheutz, M., \& Davis, G. J. (2009). Symbolic control of visual attention: Semantic constraints of the spatial distribution of attention. Attention, Perception, \& Psychophysics, 71, 363-374. doi:10.3758/APP.71.2.363

Gibson, E., Piantadosi, S., \& Fedorenko, K. (2011). Using Mechanical Turk to obtain and analyze English acceptability judgments. Language and Linguistics Compass, 5, 509-524.

Henrich, J., Heine, S. J., \& Norenzayan, A. (2010). The weirdest people in the world? Behavioral and Brain Sciences, 33, 61-83. doi:10.1017/S0140525X0999152X

Ipeirotis, P. G. (2010). Demographics of Mechanical Turk. Center for Digital Economy Research Working Papers, 10. Available at http://hdl.handle.net/2451/29585
Kemmerer, D. (2006). The semantics of space: Integrating linguistic typology and cognitive neuroscience. Neuropsychologia, 44, 16071621.

Kobayashi, H., \& Kohshima, S. (1997). Unique morphology of the human eye. Nature, 387, 767-768.

Kobayashi, H., \& Kohshima, S. (2001a). Evolution of the human eye as a device for communication. In T. Matsuzawa (Ed.), Primates origins of human cognition and behavior (pp. 383-401). Tokyo, Japan: Springer.

Kobayashi, H., \& Kohshima, S. (2001b). Unique morphology of the human eye and its adaptive meaning: Comparative studies on external morphology of the primate eye. Journal of Human Evolution, 40, 419-435.

Logan, G. D. (1995). Linguistic and conceptual control of visual spatial attention. Cognitive Psychology, 28, 103-174. doi:10.1006/cogp.1995.1004

Mason, W., \& Suri, S. (2012). Conducting behavioral research on Amazon's Mechanical Turk. Behavior Research Methods, 44, 1 23. doi:10.3758/s13428-011-0124-6

Maunsell, J. H. R., \& Van Essen, D. C. (1987). Topographic organization of the middle temporal visual area in the macaque monkey: Representational biases and the relationship to callosal connections and myeloarchitectonic boundaries. Journal of Comparative Neurology, 266, 535-555.

Myers, D. G. (1983). Social psychology. New York, NY: McGraw-Hill Education.

Nomura, M., Katahata, M., \& Hashiya, K. (2005). Visual orienting occurs asymmetrically in horizontal vs. vertical plans. Psychologia, 48, 205-217.

Ossandón, J. P., Onat, S., \& König, P. (2014). Spatial biases in viewing behavior. Journal of Vision, 14(2), 20:1-26. doi:10.1167/14.2.20

Paolacci, G., \& Chandler, J. (2014). Inside the Turk: Understanding Mechanical Turk as a participant pool. Current Directions in Psychological Science, 23, 184-188. doi:10.1177/0963721414531598

Paolacci, G., Chandler, J., \& Ipeirotis, P. G. (2010). Running experiments on Amazon Mechanical Turk. Judgment and Decision Making, 5, $411-419$.

Pauszek, J. R., \& Gibson, B. S. (2016). High spatial validity is not sufficient to elicit voluntary shifts of attention. Attention, Perception, \& Psychophysics, 78, 2110-2123. doi:10.3758/s13414-016-1097-4

Posner, M. I. (1980). Orienting of attention. Quarterly Journal of Experimental Psychology, 32, 3-25. doi:10.1080/00335558008248231

Posner, M. I., Snyder, C. R., \& Davidson, B. J. (1980). Attention and the detection of signals. Journal of Experimental Psychology: General, 109, 160-174. doi:10.1037/0096-3445.109.2.160

Robertson, L. C. (2004). Space, objects, minds, and brains. New York, NY: Psychology Press.

Schnoebelen, T., \& Kuperman, V. (2010). Using Amazon Mechanical Turk for linguistic research. Psihologija, 43, 441-464.

Sprouse, J. (2011). A validation of Amazon Mechanical Turk for the collection of acceptability judgments in linguistic theory. Behavior Research Methods, 43, 155-167. doi:10.3758/s13428-010-0039-7

Tootell, R. B. H., Switkes, E., Silverman, M. S., \& Hamilton, S. L. (1988). Functional anatomy of macaque striate cortex. II. Retinotopic organization. Journal of Neuroscience, 8, 1531-1568.

Van Essen, D. C., Newsome, W. T., \& Maunsell, J. H. (1984). The visual field representation in striate cortex of the macaque monkey: Asymmetries, anisotropies, and individual variability. Vision Research, 24, 429-448.

Witzel, J., Cornelius, S., Witzel, N., Forster, K. I., \& Forster, J. C. (2013). Testing the viability of webDMDX for masked priming experiments. Mental Lexicon, 8, 421-449.

Woods, A. T., Velasco, C., Levitan, C. A., Wan, X., \& Spence, C. (2015). Conducting perception research over the internet: A tutorial review. PeerJ, 3(e1058), 1-35. doi:10.7717/peerj.1058 